\title{
Unexpected condition in a rare disease: encephalopathy in early-onset sarcoidosis
}

\author{
Nihal Şahin ${ }^{1 \oplus}$, Sümeyra Özdemir Çiçek $^{1 \oplus}$, Ayşenur Paç Kısaarslan ${ }^{1 \oplus}$, \\ Zübeyde Gündüz ${ }^{2 \oplus}$, Muammer Hakan Poyrazoğlu' ${ }^{1 \oplus}$, Ruhan Düşünsel ${ }^{1 \oplus}$ \\ ${ }^{1}$ Department of Pediatric Rheumatology, Erciyes University Faculty of Medicine, Kayseri; ${ }^{2}$ Department of Pediatric Rheumatology, \\ Acıbadem Hospital, Kayseri, Turkey.
}

\begin{abstract}
Background. Granulomatous autoinflammatory diseases are monogenic syndromes caused by mutations in the region encoding the nucleotide-binding domain of the nucleotide-binding oligomerization domain-containing 2 gene. Blau syndrome and early-onset sarcoidosis are familial and sporadic forms of the same disease and are very rare. Many organ systems may be involved; however, neurologic involvement is infrequent. We reported a case of encephalitis in a 12-year-old girl followed with a diagnosis of early-onset sarcoidosis.
\end{abstract}

Case. The patient was diagnosed with juvenile idiopathic arthritis at 3 years of age. We considered druginduced sarcoidosis at 6 years of age with granulomatous inflammation of liver and kidney. Small joint involvement and camptodactyly developed during follow-up. M315T mutation was detected in the NOD2 gene supporting the diagnosis of early-onset sarcoidosis. The patient suffered from encephalopathy when she was under methotrexate, infliximab, and systemic steroid treatment at 12 years of age. Cerebrospinal fluid limbic encephalitis antibody panel was negative.

Conclusion. Encephalopathy is not common in Blau syndrome and early-onset sarcoidosis. The cause of encephalopathy in our patient was interpreted as autoimmune encephalitis.

Key words: Blau syndrome, early-onset sarcoidosis, encephalopathy, sarcoidosis.

Blau syndrome (BS) and early-onset sarcoidosis (EOS) are granulomatous autoinflammatory diseases defined as familial and sporadic forms of the same clinical condition, respectively. These syndromes are caused by a mutation in the nucleotide oligomerization domain 2 (NOD2) gene localized in the $16 \mathrm{q} 12$ chromosome. Clinical symptoms are characterized by the clinical triad of granulomatous dermatitis, symmetric arthritis, and recurrent uveitis with onset before 4 years of age. ${ }^{1-3}$ Non-caseating granulomatous inflammation in affected organs is a hallmark of the disease. ${ }^{4}$ Other expanded manifestations of BS/EOS are fever, pneumonitis, bronchial granulomas, hepatosplenomegaly,

$凶$ Nihal Şahin

nihal_sahin41@hotmail.com

Received 19th December 2019, revised 22nd February 2020, 5th May 2020, 9th June 2020, accepted 27th June 2020. hepatic granulomas, sialadenitis, erythema nodosum, leukocytoclastic vasculitis, transient neuropathies, arterial hypertension, pericarditis, pulmonary embolism, granulomatous glomerular and interstitial nephritis, and chronic renal failure. ${ }^{5}$ In this report, we present a patient who developed encephalitis while being followed with EOS diagnosis.

\section{Case Report}

A 12-year-old girl was diagnosed with juvenile idiopathic arthritis at 3 years of age. During that time, she had arthritis on both knees and received methotrexate (MTX) therapy. Because MTX response was inadequate, etanercept (ETN) was added. While she was on MTX and ETN treatment, at 6 years of age, she was hospitalized because of fever, neutropenia, and liver and renal failure. Multiple organ failure 
secondary to sepsis was considered. MTX and ETN were discontinued. After an antibiotic and supportive therapy, she improved in three weeks. She was hospitalized again two months later, because of fatigue and weakness. Physical examination revealed hepatomegaly and splenomegaly. Her hemoglobin level was $6.2 \mathrm{~g} / \mathrm{dl}$. The blood smear was consistent with normochromic and normocytic features. She had no evidence of nutritional and hemolytic anemia. Bone marrow aspiration showed normocellular marrow findings. She had renal failure (blood urea nitrogen $40 \mathrm{mg} / \mathrm{dl}$, creatinine $2.0 \mathrm{mg} / \mathrm{dl}$ ) without oliguria and hypertension. Proteinuria was $23 \mathrm{mg} / \mathrm{m}^{2} /$ hour. We performed liver and kidney biopsies; non-caseating granulomatous interstitial nephritis and noncaseating granulomatous inflammation of liver were detected. Urinary calcium/creatinine ratio and serum angiotensin-converting enzyme levels were normal. Tuberculin skin test was anergic, PCR for Mycobacteria of the liver tissue sample, and QuantiFERON test were negative. Since the patient was treated with ETN, we considered drug-induced sarcoidosis, and we discontinued ETN. Oral prednisolone was given and tapered. MTX was continued. Involvement of bilateral proximal interphalangeal joints and distal interphalangeal joints, boggy synovitis, and camptodactyly developed in the following year.

We reevaluated all clinical signs, and NOD2 gene analysis was performed. M513T heterozygous mutation was detected. There was consanguinity, but no family history. Thus, we diagnosed EOS and added infliximab (IFX) treatment. In the last three years, the disease activations occurred as arthritis, interstitial lung disease, interstitial nephritis, and bilateral panuveitis. Systemic steroids were added on attack periods, MTX, and IFX were continued. She was admitted to our clinic with a headache and vomiting in June 2018. These complaints were present for two weeks and were increasing, especially in the morning. She was still on MTX (15 mg/week), prednisolone (10 mg/day), and IFX (200 mg per month) treatment. High fever, high blood pressure, lethargy, diplopia, and bilateral papilledema were detected on physical examination. Laboratory examination revealed leukocytosis, increased erythrocyte sedimentation rate and C-reactive protein, and pyuria. Escherichia coli was isolated in the urine culture. Antibiotic treatment was started for urinary tract infection, and then fever resolved. Brain magnetic resonance imaging (MRI) and MRI angiography were normal. The patient underwent repeated lumbar punctures; opening pressures were $23 \mathrm{~cm} \mathrm{H}_{2} \mathrm{O}$ and $38 \mathrm{~cm}$ $\mathrm{H}_{2} \mathrm{O}$, respectively. Study of cerebrospinal fluid (CSF) revealed pleocytosis (120 leukocyte / $\mathrm{mm}^{3}$ with lymphocyte predominance), slightly elevated protein level $(35 \mathrm{mg} / \mathrm{dl})$, normal chloride, and glucose level. CSF cultures were negative. The serology for viruses and Mycobacterium tuberculosis were negative. Electroencephalography (EEG) showed delta brush waves compatible in the temporaloccipital and the parietal-occipital regions. The patient was diagnosed as autoimmune encephalitis. Cerebrospinal fluid limbic encephalitis antibody panel that included NMDA, AMPA1, AMPA2, CASPR2, LGI1, GABA $B$ antibodies were negative. Antinuclear antibody (ANA), ANA subgroups, anti-phospholipid antibodies, anti-neutrophil cytoplasmic antibodies, and direct Coombs test were negative. Although intravenous immunoglobulin was given at a dose of 400 $\mathrm{mg} / \mathrm{kg} /$ day for 5 days, lethargy, diplopia, and papilledema were not resolved. Following pulse methylprednisolone ( $1 \mathrm{~g} /$ day for 5 days) her neurological and ophthalmologic findings improved rapidly (Fig. 1). MTX, prednisolone (40 mg/day) were continued, but IFX was switched to adalimumab.

The written consent form for publishing was obtained from the patient's parents.

\section{Discussion}

We reported an EOS patient with joint involvement, granulomatous inflammation, uveitis, who developed encephalopathy in the 


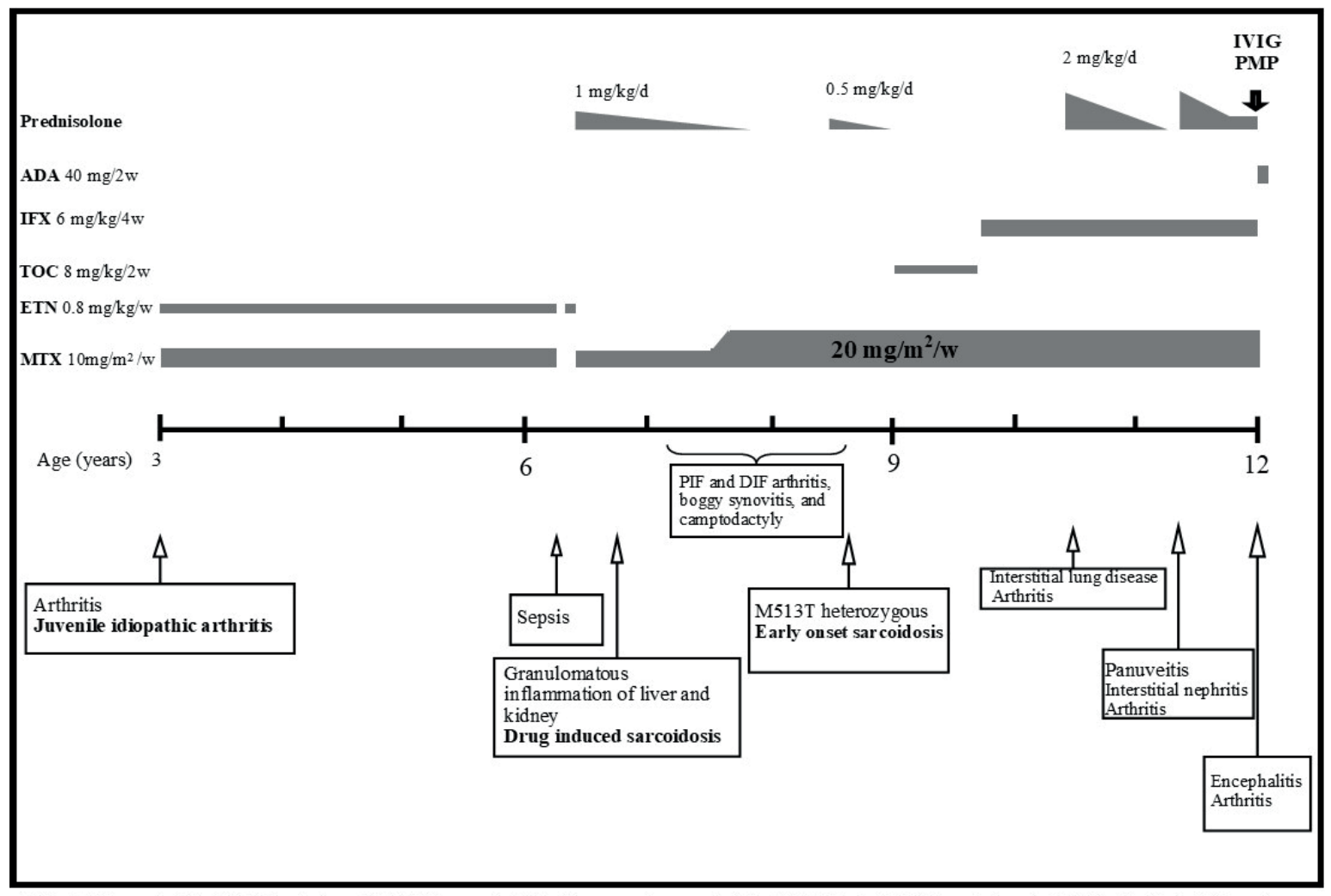

ADA: Adalimumab, DIF: Distal interphalangeal, ETN: Etanercept, IVIG: Intravenous immunoglobulin, IFX: Infliximab, MTX: methotrexate, PIF: Proximal interphalangeal, PMP: Pulse methylprednisolone, TOC: Tocilizumab

Fig. 1. Diagram of the patient's course of disease and treatments.

follow-up. Our patient was diagnosed as JIA and received JIA treatment at first because her initial finding was only arthritis. While the patient was receiving ETN and MTX, liver and kidney involvement with granulomatous inflammation in the biopsy was identified. After tuberculosis was excluded, we diagnosed drug-induced sarcoidosis as the development of sarcoidosis in patients treated with ETN has been demonstrated in previous studies. ${ }^{6}$ However, later, the diagnosis was reconsidered, because the patient's joint involvements in follow-up were boggy synovitis and camptodactyly. It is known that common joint involvements of BS/EOS are camptodactyly and tenosynovitis., ${ }^{78}$ Thus, we requested a genetic analysis. M513T heterozygous mutation was detected in the NOD2 gene. NOD2 gene localized in 16q12 chromosome encodes NOD2 protein. NOD2 protein, one of the pattern recognition receptors, is activated by muramyl dipeptide..$^{910}$ It is involved in response to bacterial infections. NOD2 protein comprises of caspase activation and recruitment domain, NOD, leucine-rich repeat domains (LRRs). While mutations in LRRs lead to Crohn's disease, BS/EOS has associated with mutations in NOD. ${ }^{11-14}$ More than $80 \%$ of patients with $\mathrm{BS} / \mathrm{EOS}$ had R334Q/W mutation. M513T is a rare NOD mutation and seen only in EOS.,15 Mutations in the domain of M513T inhibit folding, so the protein constantly remains in an active state. Active NOD2 protein has initiated pathways of NF- $\kappa B$ and stress kinase and has played a role in inflammation. ${ }^{16}$ In the functional analysis in EOS patients with M513T mutation, NF- $\kappa B$ activation increased compared to wild type ${ }^{17,18} \mathrm{NF}-\kappa \mathrm{B}$ activation is hypothesized to be responsible for granulomatous inflammation ${ }^{19}$, also, which was detected in our patient's biopsy. But we did not perform functional analysis. The clinical findings of our patient were consistent with BS/EOS, and sporadic NOD2 mutation strongly supported the diagnosis of EOS. 
The classical triad of BS/EOS is rash, uveitis, and arthritis. ${ }^{1,20}$ When our patient was diagnosed with BS/EOS, there was only arthritis from the classic triad and granulomatous inflammation in the biopsy. Uveitis, another component of the triad, developed in our patient under treatment. Similar EOS cases with uveitis developing under treatment were reported. ${ }^{21}$

Encephalopathy was a remarkable event because neurologic involvement was infrequent in BS/EOS. Neurologic involvement of EOS, central nervous system infections, and posterior reversible encephalopathy syndrome (PRES) were considered in the differential diagnosis. Hypertension is a common cause of PRES. ${ }^{22}$ But, in our patient, high blood pressure was seen in first examination and did not persist. Also, brain MRI was not compatible with PRES. Fever and elevated acute phase reactants were suggestive for infections and EOS activation; we considered urinary tract infection as the cause of fever as it resolved following treatment for urinary tract infection. In addition, CSF examination had no significant findings for central nervous system infection. EOS reports were not informative about CSF findings in neurologic involvement. But, in neurosarcoidosis, raised protein and lymphocytosis were detected. ${ }^{23}$ Therefore, we thought that CSF findings in our patient could be due to EOS. Reported neurological involvements of EOS were cranial neuropathy, papilledema, and steroid-sensitive sensorineural hearing loss. ${ }^{15,24-26}$ Emaminia et al. ${ }^{27}$ presented a BS patient who had seizures, with normal neurologic examination, cranial MRI, and EEG. They could not verify that seizures were associated with BS. In another case, seizure was detected with NOD2 mutation. This case had left middle cerebral artery and bilateral renal artery stenosis was regarded as Takayasu arteritis. ${ }^{28}$ EOS patients with M513T mutation reported in the literature had a fever, rash, arthritis, and uveitis. Also, renal calcification and erythema nodosum were seen except for the classical triad. However, there were no neurologic abnormalities in these patients with M513T mutation. ${ }^{17,18}$
We detected delta brush waves in EEG. There are case reports and case series with a delta brush pattern in patients with autoimmune encephalitis. ${ }^{24,25}$ However, Baykan et al. ${ }^{29}$ showed that this pattern was seen for various reasons in patients followed in intensive care unit. The detection of specific autoantibodies helps in the definitive diagnosis of autoimmune encephalitis and the differential diagnosis of atypical cases. The absence of autoantibodies does not exclude autoimmune encephalitis, and the positive titer does not always imply an accurate diagnosis. ${ }^{30}$

In a study, NOD2-deficient mice and wild type mice were immunized with muramyldipeptide and human serum albumin. Then the researchers assessed the antigen-specific serum immunoglobulins. Human serum albumin specific antigens were detected in wild-type mice but not in NOD2 -/- type mice. These results suggested that NOD2 activates the adaptive immune system by acting as an adjuvant receptor for antibody production directly or by increasing the production of immune-stimulatory molecules. ${ }^{31}$ Shaw et al. ${ }^{32}$ showed that resistant autoimmune encephalitis dependent on peptidoglycan in NOD1 -/-, NOD2 -/-, RIPK2 -/- mice. The authors also reported that the RIP2 signaling pathway taken part in the self-activation of the central nervous system infiltrated dendritic cells, not in the migration of $\mathrm{T}$ cells and dendritic cells. Our patient did not reveal any autoantibodies. Although our patient's clinical and EEG findings were compatible with autoimmune encephalitis, we could not be sure whether the autoimmune mechanism works in our patient or disease affect itself.

In conclusion, central nervous system involvement can be seen as encephalitis apart from classical findings of BS/EOS. We think that with the clarification of immune mechanisms in the pathogenesis of the disease, we can understand the cause of autoimmune encephalitis in our patient. 


\section{REFERENCES}

1. Blau EB. Familial granulomatous arthritis, iritis, and rash. J Pediatr 1985;107: 689-693.

2. Caso F, Rigante D, Vitale A, et al. Monogenic autoinflammatory syndromes: state of the art on genetic, clinical, and therapeutic issues. Int J Rheumatol 2013; 2013: 513782.

3. Caso F, Cantarini L, Lucherini OM, et al. Working the endless puzzle of hereditary autoinflammatory disorders. Mod Rheumatol 2014; 24: 381-389.

4. Caso F, Costa L, Rigante D, et al. Caveats and truths in genetic, clinical, autoimmune and autoinflammatory issues in Blau syndrome and early onset sarcoidosis. Autoimmun Rev 2014; 13: 1220-1229.

5. Wouters CH, Maes A, Foley KP, Bertin J, Rose CD. Blau syndrome, the prototypic auto-inflammatory granulomatous disease. Pediatr Rheumatol Online J 2014; 12: 33.

6. Verschueren K, Van Essche E, Verschueren P, Taelman V, Westhovens R. Development of sarcoidosis in etanercept-treated rheumatoid arthritis patients. Clin Rheumatol 2007; 26: 1969-1971.

7. Paç Kısaarslan A, Sözeri B, Şahin N, et al. Blau syndrome and early-onset sarcoidosis: a six case series and review of the literature. Arch Rheumatol 2020; 35: 117-127.

8. Villanueva-Mendoza C, Arellanes-Garca L, CubasLorenzo V, Jimenez-Martinez MC, Flores-Surez LF, Zenteno JC. Familial case of Blau syndrome associated with a CARD15/NOD2 mutation. Ophthalmic Genet 2010; 31: 155-158.

9. Philpott DJ, Sorbara MT, Robertson SJ, Croitoru $\mathrm{K}$, Girardin SE. NOD proteins: regulators of inflammation in health and disease. Nat Rev Immunol 2014; 14: 9-23.

10. Girardin SE, Boneca IG, Viala J, et al. Nod2 is a general sensor of peptidoglycan through muramyl dipeptide (MDP) detection. J Biol Chem 2003; 278: 8869-8872.

11. Ogura $Y$, Inohara N, Benito A, Chen FF, Yamaoka S, Núñez G. Nod2, a Nod1/Apaf-1 family member that is restricted to monocytes and activates NF-kappaB. J Biol Chem 2001; 276: 4812-4818.

12. Inohara N, Nuñez G. NODS: intracellular proteins involved in inflammation and apoptosis. Nat Rev Immunol 2003; 3: 371-382.

13. Hugot JP, Chamaillard M, Zouali $H$, et al. Association of NOD2 leucine-rich repeat variants with susceptibility to Crohn's disease. Nature 2001; 411: 599-603.
14. Ogura Y, Bonen DK, Inohara N, et al. A frameshift mutation in NOD2 associated with susceptibility to Crohn's disease. Nature 2001; 411: 603-606.

15. Wang X, Kuivaniemi H, Bonavita G, et al. CARD15 mutations in familial granulomatosis syndromes: a study of the original Blau syndrome kindred and other families with large-vessel arteritis and cranial neuropathy. Arthritis Rheum 2002; 46: 3041-3045.

16. Parkhouse R, Boyle JP, Monie TP. Blau syndrome polymorphisms in NOD2 identify nucleotide hydrolysis and helical domain 1 as signalling regulators. FEBS Lett 2014; 588: 3382-3389.

17. Takeuchi Y, Shigemura T, Kobayashi N, et al. Early diagnosis of early-onset sarcoidosis: a case report with functional analysis and review of the literature. Clin Rheumatol 2017; 36: 1189-1196.

18. Kanazawa N, Okafuji I, Kambe N, et al. Early-onset sarcoidosis and CARD15 mutations with constitutive nuclear factor-kappaB activation: common genetic etiology with Blau syndrome. Blood 2005; 105: 11951197.

19. Janssen CEI, Rose CD, De Hertogh G, et al. Morphologic and immunohistochemical characterization of granulomas in the nucleotide oligomerization domain 2-related disorders Blau syndrome and Crohn disease. J Allergy Clin Immunol 2012; 129: 1076-1084.

20. Rosé CD, Doyle TM, McIlvain-Simpson G, et al. Blau syndrome mutation of CARD15/NOD2 in sporadic early onset granulomatous arthritis. J Rheumatol 2005; 32: 373-375.

21. Okafuji I, Nishikomori R, Kanazawa N, et al. Role of the NOD2 genotype in the clinical phenotype of Blau syndrome and early-onset sarcoidosis. Arthritis Rheum 2009; 60: 242-250.

22. Tetsuka S, Ogawa T. Posterior reversible encephalopathy syndrome: a review with emphasis on neuroimaging characteristics. J Neurol Sci 2019; 404: 72-79.

23. Kidd DP. Neurosarcoidosis : clinical manifestations, investigation and treatment. Pract Neurol 2020; 20: 199-212.

24. Rosé CD, Aróstegui JI, Martin TM, et al. NOD2associated pediatric granulomatous arthritis, an expanding phenotype: study of an international registry and a national cohort in Spain. Arthritis Rheum 2009; 60: 1797-1803.

25. Aróstegui JI, Arnal C, Merino R, et al. NOD2 geneassociated pediatric granulomatous arthritis: clinical diversity, novel and recurrent mutations, and evidence of clinical improvement with interleukin-1 blockade in a Spanish cohort. Arthritis Rheum 2007; 56: 3805-3813. 
26. Jabs DA, Houk JL, Bias WB, Arnett FC. Familial granulomatous synovitis, uveitis, and cranial neuropathies. Am J Med 1985; 78: 801-804.

27. Emaminia A, Nia AE, Nabavi M, Nasab MM, Kashef S. Central nervous system involvement in Blau syndrome: a new feature of the syndrome? J Rheumatol 2007; 34: 2504-2505.

28. Inoue $Y$, Kawaguchi $Y$, Shimojo N, et al. A case of infantile Takayasu arteritis with a p.D382E NOD2 mutation: an unusual phenotype of Blau syndrome/ early-onset sarcoidosis? Mod Rheumatol 2013; 23: 837-839.

29. Baykan B, Gungor Tuncer O, Vanli-Yavuz EN, et al. Delta brush pattern is not unique to NMDAR encephalitis: evaluation of two independent longterm EEG cohorts. Clin EEG Neurosci 2018; 49: 278284.
30. Graus F, Titulaer MJ, Balu R, et al. A clinical approach to diagnosis of autoimmune encephalitis. Lancet Neurol 2016; 15: 391-404.

31. Kobayashi KS, Chamaillard M, Ogura Y, et al. Nod2-dependent regulation of innate and adaptive immunity in the intestinal tract. Science 2005; 307: 731-734.

32. Shaw PJ, Barr MJ, Lukens JR, et al. Signaling via the RIP2 adaptor protein in central nervous systeminfiltrating dendritic cells promotes inflammation and autoimmunity. Immunity 2011; 34: 75-84. 